\title{
Generación de un índice de calidad de servicio para establecimientos turísticos de alimentos y bebidas
}

\section{Generation of a service quality index for tourist food and beverage establishments}

\begin{abstract}
Ana Carola Flores Mancheno. ${ }^{1}$, Claudia Patricia Maldonado Erazo. ${ }^{2}$, Jessica Cristina Bermeo García. ${ }^{3} \&$ Catalina Margarita Verdugo Bernal. ${ }^{4}$
\end{abstract}

Recibido: 22-06-2020 /Aceptado: 20-07-2020 / Publicado: 07-08-2020

\begin{abstract}
.
DOI: https://doi.org/10.33262/concienciadigital.v3i3.1.1362

Quality represents a constant challenge that must be faced by both tourism and nontourism companies, because it depends on the evolution of the needs that the client has, for which various methods or mechanisms have been developed within the academic literature for your measurement. The present research aims to generate an index that allows evaluating the quality of the food and beverage service provided by tourist establishments in the city of Riobamba, province of Chimborazo. The methodology used corresponds to a factor analysis that synthesizes the information of 10 criteria identified in the three most representative virtual reservation platforms in the world. The main contribution that this study provides is the generation of a much more flexible measurement method that can be adapted to the variability of quality within tourist services, and that can be replicated in other sectors.
\end{abstract}

Keywords: Quality, Service, Construct, Factor analysis.

1 Escuela Superior Politécnica de Chimborazo, Facultad de Recursos Naturales. Riobamba, Ecuador., acmancheno@espoch.edu.ec

2 Escuela Superior Politécnica de Chimborazo, Facultad de Recursos Naturales. Riobamba, Ecuador., claudia.maldonado@espoch.edu.ec

3 Investigador independiente. Riobamba, Ecuador, jekabg91@gmail.com

4 Escuela Superior Politécnica de Chimborazo, Facultad de Recursos Naturales. Riobamba, Ecuador. catalina.verdugo@espoch.edu.ec 


\section{Resumen}

La calidad representa un constante desafío que debe ser afrontado por las empresas tanto turísticas como no turísticas, debido a que ésta depende de la evolución de las necesidades que el cliente posee, por lo cual dentro de la literatura académica se han desarrollado diversos métodos o mecanismos para su medición. La presente investigación tiene como objetivo generar un índice que permita evaluar la calidad del servicio de alimentos y bebidas que brindan los establecimientos turísticos de la ciudad de Riobamba, provincia de Chimborazo. La metodología empleada corresponde a un análisis factorial que sintetiza la información de 10 criterios identificados en las tres plataformas virtuales de reserva más representativas del mundo. El principal aporte que brinda este estudio es la generación de un método de medición mucho más flexible que puede adaptarse a la variabilidad de la calidad dentro de los servicios turísticos, y que puede ser replicado hacia otros sectores.

Palabras claves: Calidad, Servicio, Constructo, Análisis factorial.

\section{Introducción}

La calidad de los servicios se establece día con día en un requisito indispensable para alcanzar el posicionamiento y la competitividad deseada en el mercado (Bethencourt et al., 2005). Cuando se analiza el concepto calidad, éste siempre viene vinculado con el cliente, puesto que es él quien establece los requerimientos para determinar la calidad de lo que consume, por lo cual Cubillos y Rozo (2009) plantean que la calidad es un concepto con relación directa e inherente a la misma esencia del ser humano.

Al estar vinculada la calidad a los requerimientos establecidos por el cliente, se convierte en una condición de cambio constante y compleja de medir, puesto que las necesidades y exigencias varían, por lo que, establecer mecanismos para conseguir y comprender la calidad es un reto continuo para el sector empresarial.

Integrar la calidad dentro de los servicios que se prestan al cliente, no solo influirá en la experiencia que el cliente puede vivir, sino que también influirá en la productividad del servicio, en el ambiente laboral del personal, tanto interno como externo y en la mejora de la gestión administrativa de la empresa (Fraser et al., 2013), esto viene respaldado por Álvarez (2005) quien sostiene que el abordaje de la problemática de la calidad no solo se centra en la satisfacción de necesidades, sino que ésta corresponde a un sistema holístico que integra a toda la cadena de valor, para alcanzar así una calidad global (Castellucci, 2009)

El propósito de este estudio es generar un índice que permita evaluar la calidad del servicio de alimentos y bebidas que brindan los establecimientos turísticos de la ciudad de Riobamba, provincia de Chimborazo, calculado a través de las exigencias actuales del mercado tradicional como digital.

Este artículo se divide en cuatro secciones. Después de la introducción donde se contextualiza el tema y se plantea el objetivo, se realiza una revisión de la literatura sobre el concepto de 
calidad y sus métodos de medición. En la tercera sección se describe el enfoque metodológico utilizado para el análisis de los datos y a continuación se exponen los resultados obtenidos. Finalmente, en la última sección se presentan las principales conclusiones.

\section{a. Calidad: breve conceptualización}

Calidad es un término que tal como menciona Castellucci (2009) presenta múltiples significados que han ido variando o en su defecto evolucionando en la medida que se ha desarrollado el estudio de este tema, a pesar de ello se continua en el trabajo de su definición debido a que este concepto continua presentando dificultades de precisión.

En un breve proceso cronológico para la comprensión de la evolución del concepto hasta la actualidad se presenta la figura 1, esta analiza la calidad desde una visión macro.

Figura 1. Evolución del concepto de calidad
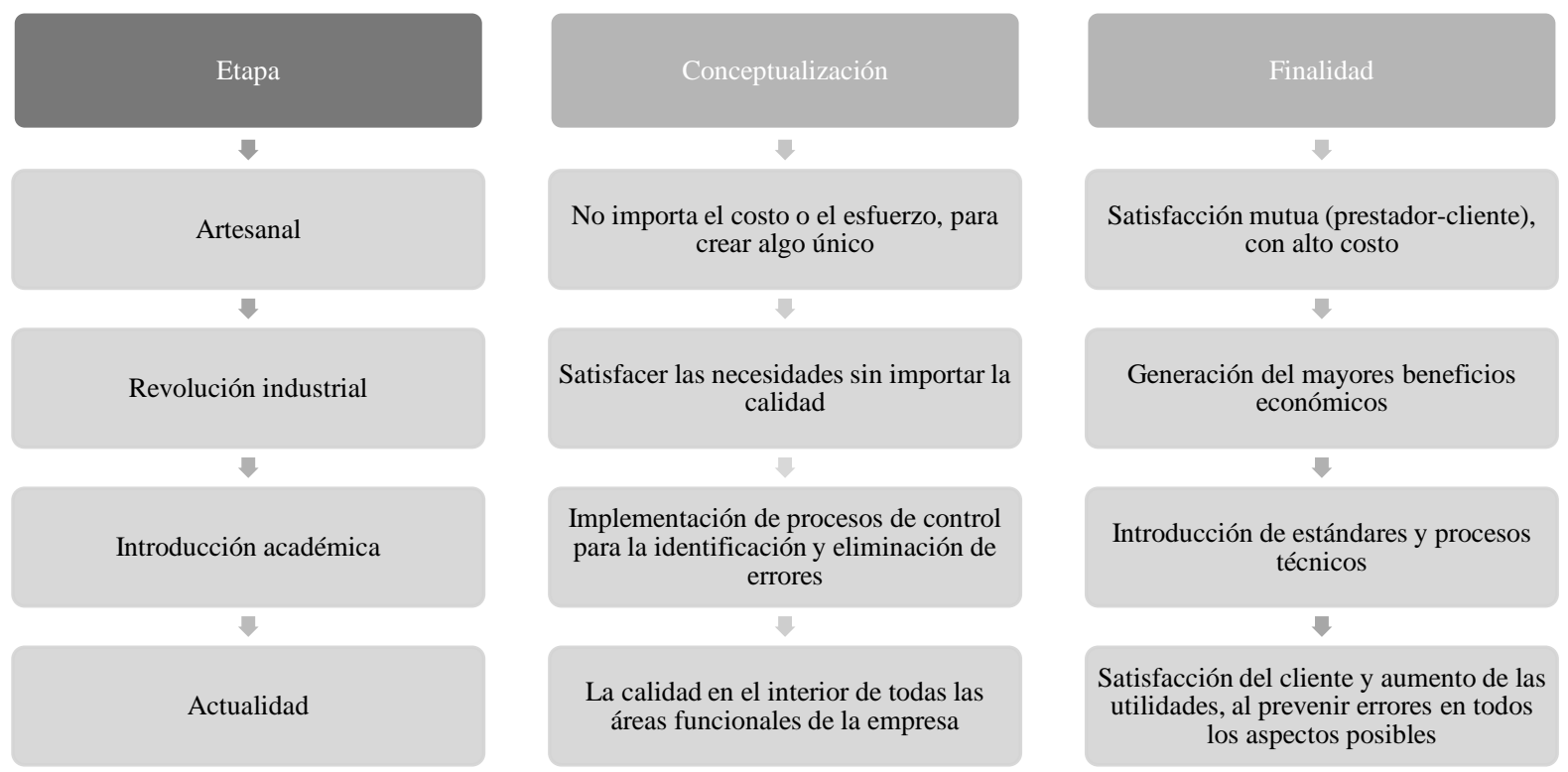

Fuente: Adaptación de Cubillos y Rozo (2009)

\section{b. Calidad y turismo}

De acuerdo con dos Anjos \& de Abreu (2009) la evaluación de la calidad se convierte en un proceso más complejo de establecer dentro de los servicios que en los productos, debido a la heterogeneidad e intangibilidad de los mismos.

De esta manera, el estudio de la calidad no ha sido algo ignorado por el turismo, debido a que el turismo es netamente un servicio (Entorno Turístico, 2020). La calidad ha sido 
analizada a distintas escalas del sistema turístico, ratificando que este concepto es algo subjetivo, pues depende de la percepción del cliente. Puntualmente, dentro del servicio de alimentos y bebidas la calidad se ha centrado en las expectativas que posee el cliente versus el servicio que recibe.

Para conseguir medirla se han generado distintas técnicas con diversos grados de complejidad, la más común es el desarrollo de un cuestionario para conocer el grado de satisfacción del cliente (Camisón et al., 2007), que generalmente se aplica a modo de feedback. Es frecuente que en este proceso se mida la calidad desde diferentes aristas, pero no se consigue una visión integral de la satisfacción alcanzada.

Entre los modelos de medición más elaborados, se encuentra el SERVQUAL que integra 5 elementos que son la fiabilidad, confianza, tangibilidad, empatía y capacidad de respuesta, es decir, se genera un instrumento a partir del cual se busca medir lo que el cliente desea o espera del servicio que consume (Parasuraman et al., 1994).

Adicionalmente, se presenta el modelo SERVPERF que mide los mismos criterios que el modelo anterior, destacando como ventajas: facilidad de aplicación, rapidez en la obtención de los resultados y mayor precisión de respuesta al evitar las ambigüedades (Chiriboga et al., 2018; Teas, 1993). Ambos métodos han sido validados en innumerables investigaciones.

A pesar de ello, los criterios propuesto por estos modelos no llegan a contener todas las necesidades actuales del cliente, que inician desde el momento en que éste decide consumir el servicio hasta cuando regresa a su hogar.

Debido a la falta de elementos que se acoplen a las nuevas exigencias, se ha optado por el diseño de un índice sintético que tome en consideración el turismo 2.0 a partir de los criterios que el turista puede valorar dentro de las plataformas de reserva de servicios. Esta consideración se efectuó debido a que, con el avance a un turismo 2.0, la calidad no solo se limita a las experiencias de familiares y amigos, sino que, integran las perspectivas de otros usuarios sin necesidad de que estén relacionados previamente (citar).

En consecuencia, la calidad del servicio al ser tan difícil medir, ésta debe ser analizada como un constructo subjetivo, generado a partir de la percepción del consumidor (Villalba, 2013). Por lo tanto, se propone la aplicación del análisis factorial (AF) como un método de reducción de datos que permite la integración de variables e identificación de factores subyacentes.

\section{Metodologia}

El estudio fue de tipo observacional, descriptivo y de corte transversal. El levantamiento de la información se realizó a través de una encuesta dirigida a los clientes de los 32 establecimientos de alimentos y bebidas que se encuentran ubicados en el centro de la ciudad y forman parte del catastro turístico municipal del año 2019. 
La fase de muestreo tuvo la duración de un mes; se seleccionaron 10 clientes de cada establecimiento obteniendo 320 encuestas, con propósito de cumplir los requerimientos de muestreo exigidos por el AF (Hair et al., 1999); los clientes seleccionados fueron escogidos de manera aleatoria bastándose como criterio de inclusión, que hayan realizado un consumo dentro del establecimiento.

La encuesta se aplicó de lunes a viernes en cuatro horarios diferentes y estuvo compuesta por diez preguntas que evaluaron la calidad percibida en los siguientes criterios o ítems: ubicación, accesibilidad, horario de apertura, servicio, ambiente, seguridad, higiene de baterías sanitarias, estándar de baterías sanitarias, higiene del personal y una evaluación general del establecimiento; los cuales se determinaron luego de efectuar un contraste entre los ítems de evaluación empleados por las tres plataformas de mayor uso para la reserva de servicios (TripAdvisor, Booking y Airbnb) vinculando de esta manera las características del turismo 2.0. Cada pregunta fue puntuada en una escala de 0 a 10 , siendo este último el que denota la máxima calidad.

\section{c. Análisis de datos}

Se inició el análisis a través de la descripción de las características de los establecimientos de alimentos y bebidas de acuerdo a su tipología y, se realizó un análisis descriptivo de la evaluación obtenida en cada uno de los diez ítems.

Posteriormente, para la integración de los ítems evaluados y obtención del índice sintético, se realizó un AF exploratorio mediante el método de componentes principales y, a través del criterio de Kaiser se identificaron el número de dimensiones o factores subyacentes, a quienes llamaremos subíndices; a cada subíndice se le asignó un nombre de acuerdo a la contribución de los ítems que lo integran.

Se generó una ecuación para el índice sintético de calidad a través de los coeficientes de ponderación y los subíndices identificados. Los coeficientes de ponderación fueron calculados como el cociente del porcentaje de variabilidad explicada por cada subíndice y el porcentaje de variabilidad total explicada por el número de factores seleccionados (Castro \& Casique, 2008).

Además, se evaluó el determinante de la matriz de correlaciones, el test de Esfericidad de Bartlett y el índice Kaiser-Meyer-Olkin (KMO) como indicadores de la pertinencia del AF.

\section{Resultados}

\section{a. Descripción de los establecimientos e ítems de evaluación}

Los 32 establecimientos de alimentos y bebidas que se encuentran en el centro de la ciudad, de acuerdo al catastro turístico municipal se clasifican como: bar, cafetería, fuente de soda y 
restaurante. En la figura 2 se muestra la distribución de los mismos, observándose que, el $66 \%$ pertenece a la tipología de restaurante.

Figura 2. Distribución de los establecimientos

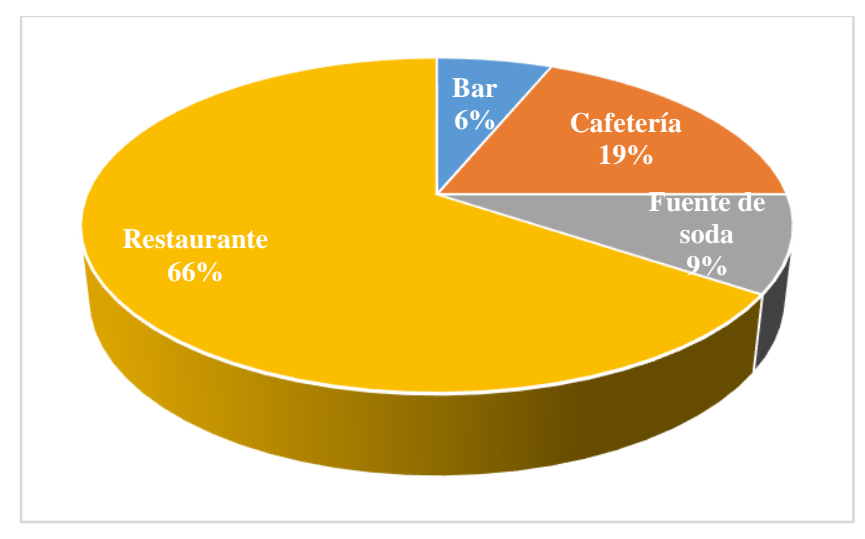

Elaborado por: Grupo de Investigación

En la tabla 1 se resume el puntaje obtenido para cada uno de los diez ítems evaluados, generados a partir de las 320 encuestas (HAIR), siendo el promedio de éstos 7,28 sobre 10 puntos. Siete de los ítems superan la puntuación promedio, destacándose la evaluación general del establecimiento con 7,85 puntos. Por otro lado, los ítems con menor evaluación son: seguridad (5,9 puntos), estándar de baterías sanitarias (6,96 puntos) e higiene de las baterías sanitarias (7,13 puntos).

La seguridad es el ítem que posee mayor variabilidad en su evaluación, ya que el coeficiente de variación alcanza un 26,05\%, mostrando con ello que, es muy diversa la percepción de calidad que tienen los clientes sobre la seguridad de los establecimientos que fueron parte de este estudio.

Tabla 1. Estadísticos descriptivos de la evaluación a los establecimientos

\begin{tabular}{rrrr}
\hline \multicolumn{1}{c}{ Estadísticos descriptivos } & \multicolumn{1}{c}{ Media } & Desv. típ. & Coef. Var. \\
\hline Ubicación (UB) & 7,42 & 0,76 & $10,24 \%$ \\
\hline Accesibilidad (AC) & 7,42 & 0,78 & $10,55 \%$ \\
\hline Higiene del personal (HP) & 7,67 & 0,90 & $11,79 \%$ \\
\hline Horario de apertura (HA) & 7,38 & 0,89 & $12,07 \%$ \\
\hline Servicio (SE) & 7,63 & 0,95 & $12,46 \%$ \\
\hline Evaluación general (EG) & 7,85 & 0,98 & $12,54 \%$ \\
\hline Higiene de baterías sanitarias (HB) & 7,13 & 1,00 & $14,03 \%$ \\
\hline Ambiente (AM) & 7,48 & 1,06 & $14,12 \%$ \\
\hline Estándar de baterías sanitarias (EB) & 6,96 & 1,21 & $17,31 \%$ \\
\hline Seguridad (SG) & 5,90 & 1,54 & $26,05 \%$ \\
Media & 7,28 & & \\
\hline
\end{tabular}

Elaborado por: Grupo de Investigación 


\section{b. Obtención del índice sintético}

\section{- Pertinencia y validez del AF}

La validez del AF como método de reducción de datos se lo realizó a partir de la evaluación del determinante de la matriz de correlaciones, el test de Esfericidad de Bartlett y el índice KMO. Cuando el determinante de la matriz de correlaciones se aproxima a cero, significa que existen variables con intercorrelaciones muy altas, siendo factible realizar el AF (Montoya, 2002).

El test de esfericidad de Bartlett prueba, si la matriz de correlaciones es una matriz identidad, es decir, prueba si las variables se encuentran correlacionadas entre sí. Si las variables no están intercorrelacionadas, entonces el test de esfericidad de Bartlett debe presentar un valor de significancia (valor p) superior al límite de 0,05. Además, el índice KMO, medido entre 0 y 1, alcanza este último valor cuando cada variable es perfectamente predicha sin error por las otras variables, considerándose como adecuado el AF si el KMO es superior a 0,80 (Hair et al., 1999).

En la tabla 2 se presentan las correlaciones existentes entre los ítems evaluados, donde 10 de ellas son mayores o iguales a 0,50. Lo deseable es que exista un número sustancial de correlaciones mayores a 0,30 (Hair et al., 1999). Aquí se observa que, la evaluación general del establecimiento se encuentra asociada principalmente con las condiciones higiénico sanitarias, ambiente y servicio del lugar. Se evidencia la factibilidad de realizar el análisis factorial ya que, el valor del determinante $(0,021)$ que se encuentra al pie de la matriz se aproxima a cero.

Tabla 2. Matriz de correlaciones de los ítems evaluados

\begin{tabular}{lcccccccccc}
\hline & $\boldsymbol{U} \boldsymbol{B}$ & $\boldsymbol{A C}$ & $\boldsymbol{H} \boldsymbol{A}$ & $\boldsymbol{S} \boldsymbol{E}$ & $\boldsymbol{A} \boldsymbol{M}$ & $\boldsymbol{S} \boldsymbol{G}$ & $\boldsymbol{H B}$ & $\boldsymbol{E B}$ & $\boldsymbol{H P}$ & $\boldsymbol{E} \boldsymbol{G}$ \\
\hline UB & 1,00 & & & & & & & & & \\
\hline AC & 0,30 & 1,00 & & & & & & & \\
\hline HA & 0,07 & 0,30 & 1,00 & & & & & & \\
\hline SE & 0,38 & 0,25 & 0,19 & 1,00 & & & & & \\
\hline AM & 0,45 & 0,13 & 0,16 & 0,44 & 1,00 & & & & & \\
\hline SG & 0,09 & 0,21 & 0,23 & 0,29 & 0,13 & 1,00 & & & & \\
\hline HB & 0,36 & 0,38 & 0,36 & 0,43 & 0,47 & 0,35 & 1,00 & & & \\
\hline EB & 0,32 & 0,39 & 0,23 & $\mathbf{0 , 5 1}$ & $\mathbf{0 , 5 0}$ & 0,32 & $\mathbf{0 , 6 7}$ & 1,00 & & \\
\hline HP & 0,28 & 0,28 & 0,25 & 0,47 & 0,49 & 0,29 & $\mathbf{0 , 5 3}$ & $\mathbf{0 , 5 5}$ & 1,00 & \\
\hline EG & 0,45 & 0,27 & 0,11 & $\mathbf{0 , 5 5}$ & $\mathbf{0 , 6 3}$ & 0,21 & $\mathbf{0 , 5 3}$ & $\mathbf{0 , 5 8}$ & $\mathbf{0 , 6 8}$ & 1,00 \\
\hline
\end{tabular}

Determinante $=0,021$

A partir del test de Esfericidad de Bartlett (tabla 3) se corrobora la validez de la aplicación del AF, debido a que la significancia obtenida es 0,000 . En cuanto al índice $\operatorname{KMO}(0,86)$ 
comprueba el grado de relación conjunta entre los ítems, considerándose que éstos pueden ser predecibles a partir de los demás.

Tabla 3. KMO y prueba de Bartlett

\begin{tabular}{llr}
\hline Medida de adecuación muestral de Kaiser-Meyer-Olkin & 0,860 \\
\hline $\begin{array}{l}\text { Prueba de esfericidad de } \\
\text { Bartlett }\end{array}$ & Chi-cuadrado aproximado & 1218,461 \\
\cline { 2 - 3 } & gl & 45 \\
\cline { 2 - 3 } & Sig. & 0,000 \\
\hline
\end{tabular}

Con la verificación de la pertinencia y validez del análisis factorial, se procede con la extracción de los factores subyacentes a través de la agrupación de los 10 ítems evaluados.

- Extracción de los factores

Para seleccionar el número de factores que explicaran los 10 ítems, se utilizó el criterio de Kaiser, que consiste en conservar aquellos factores cuyo autovalor asociado sea mayor que 1 (Hair et al., 1999). En la figura 3 se observa que los dos primeros componentes tienen autovalores mayores a 1 , por lo que se extraen dos factores.

Figura 3. Diagrama de sedimentación

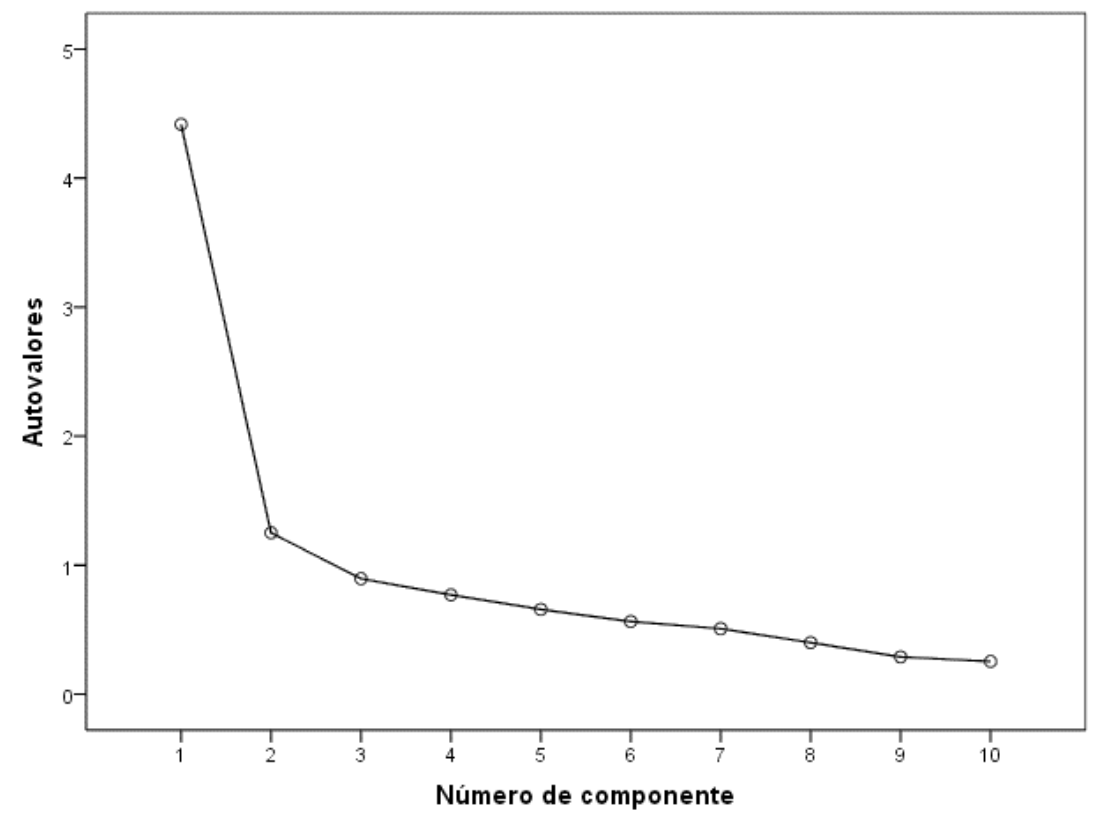

En la tabla XX se detallan los autovalores iniciales de cada componente, en ella se evidencia que los dos primeros componentes tienen autovalores mayores a 1 y que en conjunto explican el 56,66\% de la variabilidad de los 10 ítems, de tal manera que, se identifican dos factores 
subyacentes que resumen dicha información.

Tabla 4. Autovalores y varianza total explicada

\begin{tabular}{ccccccr}
\hline \multirow{2}{*}{ Componente } & \multicolumn{2}{c}{ Autovalores iniciales } & \multicolumn{3}{c}{ Suma de las saturaciones al cuadrado de } \\
& \cline { 2 - 7 } Ta rotación & \\
& Total & $\begin{array}{c}\text { \% de la } \\
\text { varianza }\end{array}$ & $\begin{array}{c}\text { \% } \\
\text { acumulado }\end{array}$ & Total & $\begin{array}{c}\text { \% de la } \\
\text { varianza }\end{array}$ & $\begin{array}{c}\text { \% } \\
\text { acumulado }\end{array}$ \\
\hline 1 & 4,416 & 44,161 & 44,161 & 3,572 & 35,718 & 35,718 \\
\hline 2 & 1,251 & 12,505 & 56,666 & 2,095 & 20,948 & 56,666 \\
\hline 3 & 0,895 & 8,951 & 65,617 & & & \\
\hline 4 & 0,77 & 7,697 & 73,313 & & \\
\hline 5 & 0,657 & 6,569 & 79,882 & & \\
\hline 6 & 0,563 & 5,627 & 85,509 & & \\
\hline 7 & 0,508 & 5,076 & 90,586 & & & \\
\hline 8 & 0,399 & 3,994 & 94,58 & & & \\
\hline 9 & 0,288 & 2,885 & 97,465 & & & \\
\hline 10 & 0,254 & 2,535 & 100 & & & \\
\hline
\end{tabular}

Método de extracción: Análisis de Componentes principales

En la tabla 5, se presenta las cargas factoriales rotadas utilizando el método Varimax, logrando así que, la identificación de los factores subyacentes sea más sencilla. Las cargas factoriales, representan la correlación que existe entre cada ítem y el factor, lo deseable es que cada ítem contribuya en la formación de un solo factor, por lo que, idealmente los valores mayores a 0,5 se consideran adecuados (Pérez, 2004). De acuerdo a lo indicado, se generan dos factores a quienes se los denominará subíndices los mismos que se encuentran compuestos de la siguiente manera:

- Subíndice de Condición del Servicio (SCS): Está compuesto por siete ítems los cuales son: evaluación general, ambiente, higiene del personal, servicio, estándar de baterías sanitarias, ubicación e higiene de baterías sanitarias.

- Subíndice de Factores de Acceso (SFA): Compuesto por tres ítems: horarios de apertura, seguridad y accesibilidad.

Tabla 5. Matriz de componentes rotados

\begin{tabular}{lrrr}
\hline & Ítems & \multicolumn{2}{c}{ Componentes o factores } \\
\cline { 2 - 4 } & SCS & SFA \\
\hline EG & $\mathbf{0 , 8 6 3}$ & 0,138 \\
\hline AM & $\mathbf{0 , 8 0 7}$ & 0,030 \\
\hline HP & $\mathbf{0 , 6 8 1}$ & 0,358 \\
\hline SE & $\mathbf{0 , 6 6 6}$ & 0,266 \\
\hline
\end{tabular}




\section{ISSN: 2600-5859}

Vol. 3, N³.1, p. 44-57, agosto, 2020

\begin{tabular}{lcr}
\hline EB & $\mathbf{0 , 6 6 2}$ & 0,461 \\
\hline UB & $\mathbf{0 , 6 5 7}$ & 0,004 \\
\hline HB & $\mathbf{0 , 5 8 3}$ & 0,564 \\
\hline HA & $-0,009$ & $\mathbf{0 , 7 4 7}$ \\
\hline SG & 0,119 & $\mathbf{0 , 6 3 8}$ \\
\hline AC & 0,210 & $\mathbf{0 , 6 1 6}$ \\
\hline
\end{tabular}

Método de extracción: Análisis de componentes principales.

Método de rotación: Normalización Varimax con Kaiser.

a. La rotación ha convergido en 3 iteraciones.

- Ecuación del índice sintético

Para la integración de los dos subíndices y de sus respectivos ítems en un solo indicador, primero se calculan los coeficientes de ponderación, dividiendo el porcentaje de variabilidad explicada por cada subíndice para el porcentaje de variabilidad total explicada por los dos factores extraídos.

$$
\begin{gathered}
\omega=\frac{\% \text { varianza }}{\% \text { acumulado }} \\
\omega_{1}=\frac{35,718}{56,666}=0,63 \\
\omega_{2}=\frac{20,948}{56,666}=0,37
\end{gathered}
$$

Luego, se calculan los subíndices, sumando los valores de los ítems dentro de cada factor y dividiendo este valor para la puntuación máxima que podría ser alcanzada. En el caso del primer subíndice, se suman las puntuaciones de los 7 ítems que lo conforman y se divide para 70; de manera análoga se procede con el segundo subíndice, es decir, se suman las puntuaciones de los 3 ítems que lo integran y se divide para 30 . Como cada establecimiento tiene la evaluación a través de 10 encuestas, se promedian estos valores obteniendo una sola puntuación por establecimiento.

De esta manera, el Índice de Calidad del Servicio (ICS) queda integrado por la ecuación:

$$
I C S=0,63 * S C S+0,37 * S F A
$$


Obteniéndose un índice de calidad cuyo rango de evaluación está definido entre 0 y 1.

En la tabla 6 se observa a los establecimientos ordenados de manera descendente de acuerdo a la valoración de calidad alcanzada, misma que se calculó a través de las puntuaciones de los subíndices. Aquí se aprecia que, tanto los restaurantes (REST) como bares, cafeterías y fuentes de soda (BCF) tienen diversas puntuaciones, las mismas que fluctúan entre 0,55 y 0,87 sobre 1 punto, con una media de 0,72 y desviación estándar de 0,06.

Tabla 6. Composición de los subíndices e índice de calidad del servicio por establecimiento

\begin{tabular}{|c|c|c|c|}
\hline Tipo de establecimiento & SCS & SFA & ICS \\
\hline REST & 0,88 & 0,84 & 0,87 \\
\hline REST & 0,83 & 0,81 & 0,82 \\
\hline $\mathrm{BCF}$ & 0,83 & 0,77 & 0,81 \\
\hline REST & 0,81 & 0,79 & 0,80 \\
\hline REST & 0,80 & 0,78 & 0,79 \\
\hline REST & 0,77 & 0,75 & 0,76 \\
\hline REST & 0,78 & 0,72 & 0,76 \\
\hline $\mathrm{BCF}$ & 0,76 & 0,75 & 0,76 \\
\hline REST & 0,77 & 0,72 & 0,75 \\
\hline $\mathrm{BCF}$ & 0,80 & 0,65 & 0,74 \\
\hline REST & 0,80 & 0,65 & 0,74 \\
\hline REST & 0,80 & 0,64 & 0,74 \\
\hline REST & 0,82 & 0,60 & 0,74 \\
\hline $\mathrm{BCF}$ & 0,79 & 0,65 & 0,74 \\
\hline REST & 0,76 & 0,70 & 0,74 \\
\hline REST & 0,79 & 0,64 & 0,73 \\
\hline REST & 0,76 & 0,69 & 0,73 \\
\hline REST & 0,78 & 0,64 & 0,73 \\
\hline REST & 0,78 & 0,63 & 0,72 \\
\hline $\mathrm{BCF}$ & 0,71 & 0,71 & 0,71 \\
\hline REST & 0,70 & 0,71 & 0,70 \\
\hline $\mathrm{BCF}$ & 0,69 & 0,72 & 0,70 \\
\hline $\mathrm{BCF}$ & 0,68 & 0,71 & 0,69 \\
\hline REST & 0,68 & 0,71 & 0,69 \\
\hline REST & 0,71 & 0,65 & 0,69 \\
\hline REST & 0,67 & 0,71 & 0,68 \\
\hline REST & 0,67 & 0,69 & 0,68 \\
\hline $\mathrm{BCF}$ & 0,67 & 0,68 & 0,67 \\
\hline REST & 0,68 & 0,63 & 0,66 \\
\hline $\mathrm{BCF}$ & 0,67 & 0,63 & 0,66 \\
\hline $\mathrm{BCF}$ & 0,63 & 0,60 & 0,62 \\
\hline $\mathrm{BCF}$ & 0,56 & 0,52 & 0,55 \\
\hline
\end{tabular}

\section{Conclusiones}

- Los servicios presentan una alta complejidad para la medición de la calidad, hecho que no es ajeno a los servicios turísticos, debido a la alta variabilidad de la percepción del 
cliente, factor por el cual se establece como uno de los cinco pilares de trabajo del MINTUR asegurar la calidad en la prestación de servicios turísticos para con ello, generar una experiencia turística que cumpla con las exceptivas del consumidor y que beneficie a la cadena de valor y los destinos turísticos del país.

- Con respecto al análisis efectuado, se ha podido validar que éste representa una herramienta de gran utilidad puesto que ha permitido sintetizar la información de diez ítems en un solo índice, con lo cual, no se descarta que la generación de información a mayor escala pueda ser traducida a información de fácil comprensión.

- Un 68,75 de los establecimientos evaluados obtuvieron una puntuación de al menos 0,70 sobre 1 punto, evidenciando que una parte representativa de ellos posee una calidad aceptable en el servicio prestado. Es necesario precisar que, la seguridad es el ítem que alcanzó la menor evaluación en los establecimientos, siendo un elemento a mejorar dentro de este servicio turístico.

- Finalmente, se recomienda ampliar el levantamiento de la información a los fines de semanas y hacer una comparación con el presente análisis, con fin de determinar si existe diferencias significativas en la percepción de calidad abarcado el tipo de establecimiento y segmento de mercado.

\section{Referencias bibliográficas.}

Alvarez, A. (2005). La contribución del turismo al desarrollo integral de las sociedades receptoras. Aspectos teórico-metodológicos. Revista Política y Sociedad, 42(1), 57-84.

Bethencourt, M., Díaz, F., González, M. O., \& Sánchez, J. (2005). La medición de la calidad de los servicios prestados por los destinos turísticos: el caso de las isla de La Palma. Pasos. Revista de Turismo y Patrimonio Cultural, 3(2), 265-272. https://doi.org/https://doi.org/10.25145/j.pasos.2005.03.021

Camisón, C., Cruz, S., \& González, T. (2007). Gestión de la calidad: conceptos, enfoques, modelos y sistenas. Pearson Educación.

Castellucci, D. (2009). La calidad y los servicios en destinos turísticos maduros. Aportes y Transferencias, 13(2), 29-44.

Castro, R., \& Casique, I. (2008). Violencia de género en las parejas mexicanas. Análisis de resultados de la Encuesta Nacional sobre la dinámica de las relaciones en los hogares 2006.

Chiriboga, E., Guamán, Y., Pérez, M., Hidalgo, J., \& Mora, F. (2018). Calidad del servicio y su impacto en el turismo cultural: estudio de caso Museo Municipal. Revista Espacios, 39(19), 9-33.

Cubillos, M., \& Rozo, D. (2009). El concepto de calidad: Historia, evolución e importancia para la competitividad. Revista de La Universidad de La Salle, 4, 80-99. https://ciencia.lasalle.edu.co/cgi/viewcontent.cgi?article $=1170 \&$ context $=$ ruls 
dos Anjos, S., \& de Abreu, A. F. (2009). La medición de la calidad de servicio: una aplicación en empresas hoteleras. Revista Europea de Dirección y Economía de La Empresa, 18(2), $175-186$.

Entorno Turístico. (2020). La calidad en el turismo. https://www.entornoturistico.com/lacalidad-en-el-turismo/

Fraser, K., Watanabe, C., \& Hvolby, H.-H. (2013). Commitment to service quality in automative dealerships: results from an australian pilot study. Journal of Services, 13(1), $7-29$.

Hair, J., Anderson, R., Tatham, R., \& Black, W. (1999). Análisis Multivariante. Prentice Hall International.

Montoya, O. (2002). Analisis de Datos Multivariantes. McGraw-hill.

Parasuraman, A., Zeithaml, V., \& Berry, L. (1994). Reassessment of Expectations as a Comparison Standard in Measuring Service Quality : Implications for Further Research. Journal of Marketing, 58(1), 111-124.

Pérez, C. (2004). Técnicas de Análisis Multivariante de Datos. Pearson Prentice Hall.

Teas, R. (1993). Expectations, Performance Evaluation, and Consumers' Perceptions of Quality. Journal of Marketing, 57(4), 18-34. https://doi.org/10.2307/1252216

Villalba, C. (2013). La calidad del servicio: un recorrido histórico conceptual, sus modelos más representativos y su aplicación en las universidades. REvisa Punto de Vista, IV(7), $51-72$.

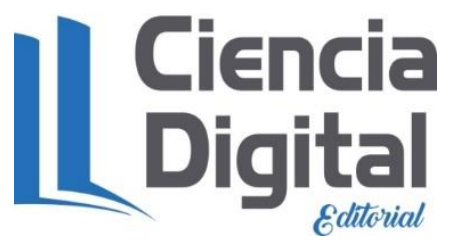




\section{PARA CITAR EL ARTÍCULO INDEXADO.}

Flores Mancheno , A. C., Maldonado Erazo, C. P., Bermeo García, J. C., \& Verdugo Bernal , C. M. (2020). Generación de un índice de calidad de servicio para establecimientos turísticos de alimentos y bebidas. ConcienciaDigital, 3(3.1), 44-57. https://doi.org/10.33262/concienciadigital.v3i3.1.1362

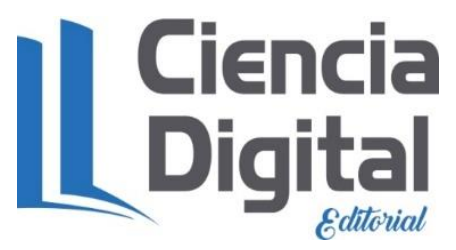

El artículo que se publica es de exclusiva responsabilidad de los autores y no necesariamente reflejan el pensamiento de la Revista Conciencia Digital.

El artículo queda en propiedad de la revista y, por tanto, su publicación parcial y/o total en otro medio tiene que ser autorizado por el director de la Revista Conciencia Digital.

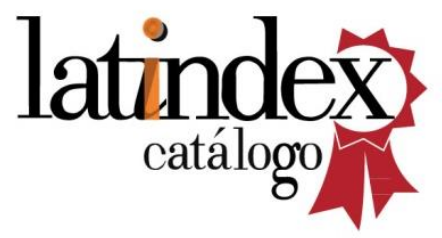

